\title{
Confinement-deconfinement phase transition for heavy quarks in a soft wall holographic QCD model
}

\author{
Yi Yang and Pei-Hung Yuan \\ Department of Electrophysics, National Chiao Tung University, \\ Hsinchu, R.O.C. \\ Institute of Physics, National Chiao Tung University, \\ Hsinchu, R.O.C. \\ E-mail: yiyang@mail.nctu.edu.tw, phy.pro.phy@gmail.com
}

ABSTRACT: We study confinement-deconfinement phase transition for heavy quarks in a soft wall holographic QCD model. We consider a black hole background in an EinsteinMaxwell-scalar system and add probe open strings to the background. Combining the various configurations of the open strings and the phase structure of the black hole background itself, we obtain the confinement-deconfinement phase diagram for heavy quarks in the holographic QCD model.

KEYwORDS: Phase Diagram of QCD, Gauge-gravity correspondence, Wilson, 't Hooft and Polyakov loops

ARXiv EPRINT: 1506.05930 


\section{Contents}

1 Introduction 1

2 Einstein-Maxwell-scalar system 3

2.1 Background 3

2.2 Phase structure of the background 5

3 Open strings in the background $\quad 8$

3.1 Wilson loop $\quad 9$

$\begin{array}{lll}3.2 & \text { Configurations of open strings } & 10\end{array}$

$\begin{array}{lll}3.3 & \text { Heavy quark potential } & 12\end{array}$

4 Phase diagram $\quad \mathbf{1 5}$

$\begin{array}{lll}4.1 & \text { Confinement-deconfinement phase diagram } & 16\end{array}$

$\begin{array}{lll}4.2 & \text { Meson melting in hot plasma } & 17\end{array}$

$\begin{array}{llr}5 & \text { Conclusion } & 18\end{array}$

\section{Introduction}

Confinement-deconfinement phase transition is an important and challenging problem in QCD. Near the phase transition region, the interaction becomes very strong so that the conventional perturbation method of QFT does not work. For a long time, lattice QCD has been the only method to study strong interacted QCD. Although lattice QCD works well for zero density, it encounters the sign problem when considering finite quark density. See $[1,2]$ for a review of the current status of lattice QCD. Recently, using the idea of AdS/CFT duality from string theory, one is able to study QCD in the strongly coupled region by studying its weakly coupled dual gravitational theory, i.e. holographic QCD [319]. In [20], we considered a Einstein-Maxwell-scalar system and studied its holographic dual QCD model. We obtained a family of analytic black hole solutions by the potential reconstruction method. By studying the thermodynamics of the black hole backgrounds, we found a phase transition between two black holes with different size. We interpreted this black hole to black hole phase transition as the confinement-deconfinement phase transition of heavy quarks in the dual holographic QCD model.

On the other hand, the heavy quark potential is an important observable relevant to confinement. It has been measured in great detail in lattice simulations [21] and the results remarkably agree with the Cornell potential [22]

$$
V(r)=-\frac{\kappa}{r}+\sigma_{s} r+C
$$


which is dominant by Coulomb potential at short distances and by linear potential at large distances with the coefficient $\sigma_{s}$ defined as string tension. In QCD, the heavy quark potential can be read off from the expectation value of the Wilson loop along a time-like closed path $C$,

$$
\langle W(C)\rangle \sim e^{-t V(r)} .
$$

In string/gauge duality, the expectation value of the Wilson loop is given by [23]

$$
\langle W(C)\rangle=\int D X e^{-S_{\mathrm{NG}}},
$$

where $S_{\mathrm{NG}}$ is the string world-sheet action bounded by the loop $C$ at the boundary of an AdS space. In [24-35], a probe open string in an AdS background was considered. The two ends of the open string are attached to the boundary of AdS background and behave as a quark-antiquark pair. Thus the open string could be interpreted as a bound state, i.e. meson state, in QCD. By studying the dynamics of the open string, the expectation value of the Wilson loop can be obtained, so as the heavy quark potential. From the behavior of the heavy quark potential, one is able to study the process that an open string breaks to two open strings with their two ends attaching to the AdS boundary and the black hole horizon, respectively. This string breaking phenomenon describes how a meson melts to a pair of free quark and antiquark in its dual QCD.

In this work, we put probe open strings in the background obtained in [20]. We study the dynamics of the open strings to obtain the expectation value of the Wilson loop as well as the heavy quark potential. In [20], various black hole phases for different temperatures have been obtained. In this work, we found three open string configurations for the various black hole phases as in figure 1. According to AdS/QCD duality, these different open string configurations correspond to the confinement and deconfinement phases in QCD, respectively. This supports our preferred interpretation that the black hole to black hole phase transition in the bulk corresponds to the confinement-deconfinement phase transition of heavy quarks in the dual holographic QCD in [20]. Nevertheless, we found that the phase transition temperatures obtained from the black hole phases and the open string configurations are not exact the same. In fact, we will argue that neither the black hole phases nor the string configurations alone could explain the full phase structure of the confinement-deconfinement phase transition in QCD. The string configurations tells us that whether the system is in confinement or deconfinement phase, while the black hole phase transition determines the location of the phase boundary. By combining the two effects together in this paper, we find a more natural picture to describe the phase diagram of the confinement-deconfinement transition for the heavy quarks in QCD. Furthermore, in the deconfinement phase, we also study the meson melting process by studying the process of an open string breaking to two open strings.

The paper is organized as follows. In section 2, we consider an Einstein-Maxwell-scalar system. We review how to get the analytic solutions in [20] by potential reconstruction method and study the phase structure in the these backgrounds. In section 3, we add probe open strings in our black hole background to study their various configurations. We calculate the expectation value of the Wilson loop and study the heavy quark potential. 


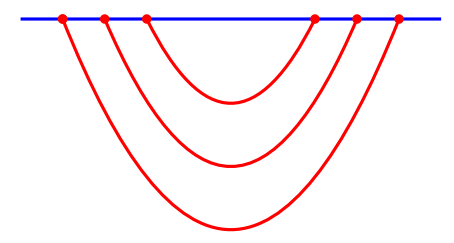

(a) no $\mathrm{BH}$

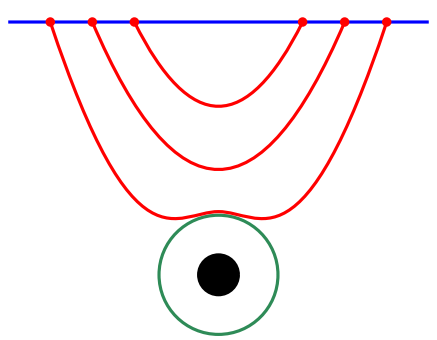

(b) small $\mathrm{BH}$

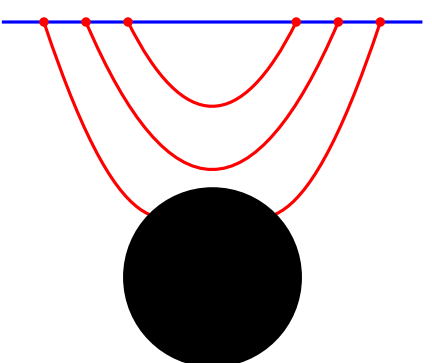

(c) large $\mathrm{BH}$

Figure 1. Three configurations for open strings in a black hole. There is no black hole in case (a), open strings are always connected with their two ends on the AdS boundary. In the small black hole case (b), open strings can not exceed a certain distance from the boundary and are still connected with their two ends on the AdS boundary. In the large black hole case (c), the open strings with their two ends far away enough will break to two open strings with their two ends attaching the AdS boundary and the black hole horizon, respectively.

In section 4 , by combining the background phase structure and the open string breaking effect, we obtain the phase diagram for confinement-deconfinement transition. We further study the meson melting process in the deconfinement phase. We conclude our result in section 4 .

\section{Einstein-Maxwell-scalar system}

In this section, we review the black hole solution and its phase structure obtained in [20].

\subsection{Background}

We consider a 5-dimensional Einstein-Maxwell-scalar system with probe matters. The action of the system has two parts, the background part and the matter part,

$$
S=S_{b}+S_{m}
$$

In Einstein frame, the background action includes a gravity field $g_{\mu \nu}$, a Maxwell field $A_{\mu}$ and a neutral scalar field $\phi$, while the matter action includes a massless gauge fields $A_{\mu}^{V}$, which we will treat as probe, describing the degrees of freedom of vector mesons on the 4d boundary,

$$
\begin{aligned}
S_{b} & =\frac{1}{16 \pi G_{5}} \int d^{5} x \sqrt{-g}\left[R-\frac{f(\phi)}{4} F^{2}-\frac{1}{2} \partial_{\mu} \phi \partial^{\mu} \phi-V(\phi)\right], \\
S_{m} & =-\frac{1}{16 \pi G_{5}} \int d^{5} x \sqrt{-g} \frac{f(\phi)}{4} F_{V}^{2},
\end{aligned}
$$

where $G_{5}$ is the coupling constant for the gauge field strength $F_{\mu \nu}=\partial_{\mu} A_{\nu}-\partial_{\nu} A_{\mu}, f(\phi)$ is the gauge kinetic function associated to the Maxwell field $A_{\mu}$ and $V(\phi)$ is the potential of the scalar field $\phi$. 
The equations of motion can be derived from the above action as

$$
\begin{array}{r}
\nabla^{2} \phi=\frac{\partial V}{\partial \phi}+\frac{1}{4} \frac{\partial f}{\partial \phi}\left(F^{2}+F_{V}^{2}\right), \quad \nabla_{\mu}\left[f(\phi) F^{\mu \nu}\right]=0, \quad \nabla_{\mu}\left[f(\phi) F_{V}^{\mu \nu}\right]=0 \\
R_{\mu \nu}-\frac{1}{2} g_{\mu \nu} R=\frac{f(\phi)}{2}\left(F_{\mu \rho} F_{\nu}^{\rho}-\frac{1}{4} g_{\mu \nu} F^{2}\right)+\frac{1}{2}\left[\partial_{\mu} \phi \partial_{\nu} \phi-\frac{1}{2} g_{\mu \nu}(\partial \phi)^{2}-g_{\mu \nu} V\right] .
\end{array}
$$

To solve the background of the Einstein-Maxwell-scalar system, we first turn off the probe gauge field $A_{\mu}^{V}$ and consider the ansatz for the metric, scalar field and Maxwell field as,

$$
\begin{aligned}
d s^{2} & =\frac{e^{2 A(z)}}{z^{2}}\left[-g(z) d t^{2}+\frac{d z^{2}}{g(z)}+d \vec{x}^{2}\right], \\
\phi & =\phi(z), \quad A_{\mu}=A_{t}(z)
\end{aligned}
$$

which leads to the following equations of motion for the background fields,

$$
\begin{aligned}
\phi^{\prime \prime}+\left(\frac{g^{\prime}}{g}+3 A^{\prime}-\frac{3}{z}\right) \phi^{\prime}+\left(\frac{z^{2} e^{-2 A} A_{t}^{\prime 2} f_{\phi}}{2 g}-\frac{e^{2 A} V_{\phi}}{z^{2} g}\right) & =0, \\
A_{t}^{\prime \prime}+\left(\frac{f^{\prime}}{f}+A^{\prime}-\frac{1}{z}\right) A_{t}^{\prime} & =0, \\
A^{\prime \prime}-A^{\prime 2}+\frac{2}{z} A^{\prime}+\frac{\phi^{\prime 2}}{6} & =0, \\
g^{\prime \prime}+\left(3 A^{\prime}-\frac{3}{z}\right) g^{\prime}-e^{-2 A} z^{2} f A_{t}^{\prime 2} & =0, \\
A^{\prime \prime}+3 A^{\prime 2}+\left(\frac{3 g^{\prime}}{2 g}-\frac{6}{z}\right) A^{\prime}-\frac{1}{z}\left(\frac{3 g^{\prime}}{2 g}-\frac{4}{z}\right)+\frac{g^{\prime \prime}}{6 g}+\frac{e^{2 A} V}{3 z^{2} g} & =0 .
\end{aligned}
$$

To solve the above equations of motion, we need to specify the following boundary and physical conditions:

1. Near the boundary $z \rightarrow 0$, we require the metric in string frame to be asymptotic to $A d S_{5}$

2. Near the horizon $z=z_{H}$, we put the regular condition $A_{t}\left(z_{H}\right)=g\left(z_{H}\right)=0$;

3. The vector meson spectrum should satisfy the linear Regge trajectories at zero temperature and zero density [36].

With the above conditions, the equations of motion (2.8)-(2.12) can be analytically solved as

$$
\begin{aligned}
\phi^{\prime}(z) & =\sqrt{-6\left(A^{\prime \prime}-A^{2}+\frac{2}{z} A^{\prime}\right)}, \\
A_{t}(z) & =\mu \frac{e^{c z^{2}}-e^{c z_{H}^{2}}}{1-e^{c z_{H}^{2}}}
\end{aligned}
$$




$$
\begin{aligned}
& g(z)=1+\frac{1}{\int_{0}^{z_{H}} y^{3} e^{-3 A} d y} \times \\
& \times\left[\frac{2 c \mu^{2}}{\left(1-e^{c z_{H}^{2}}\right)^{2}}\left|\begin{array}{cc}
\int_{0}^{z_{H}} y^{3} e^{-3 A} d y & \int_{0}^{z_{H}} y^{3} e^{-3 A} e^{c y^{2}} d y \\
\int_{z_{H}}^{z} y^{3} e^{-3 A} d y & \int_{z_{H}}^{z} y^{3} e^{-3 A} e^{c y^{2}} d y
\end{array}\right|-\int_{0}^{z} y^{3} e^{-3 A} d y\right] \\
& V(z)=-3 z^{2} g e^{-2 A}\left[A^{\prime \prime}+3 A^{\prime 2}+\left(\frac{3 g^{\prime}}{2 g}-\frac{6}{z}\right) A^{\prime}-\frac{1}{z}\left(\frac{3 g^{\prime}}{2 g}-\frac{4}{z}\right)+\frac{g^{\prime \prime}}{6 g}\right]
\end{aligned}
$$

where $\mu \equiv A_{t}(0)$ is defined as chemical potential.

The solution (2.13)-(2.16) depends on the warped factor $A(z)$. The choice of $A(z)$ is arbitrary provided it satisfies the boundary conditions. To be concrete, we fix the warped factor $A(z)$ in our solution in a simple form as

$$
A(z)=-\frac{c}{3} z^{2}-b z^{4}
$$

where the parameters $b$ and $c$ will be determined later.

\subsection{Phase structure of the background}

With the background (2.6), one can calculate the Hawking-Bekenstein entropy

$$
s=\frac{e^{3 A\left(z_{H}\right)}}{4 z_{H}^{3}}
$$

and the Hawking temperature

$$
T=\frac{z_{H}^{3} e^{-3 A\left(z_{H}\right)}}{4 \pi \int_{0}^{z_{H}} y^{3} e^{-3 A} d y}\left[1-\frac{2 c \mu^{2}\left(e^{\left.c z_{H}^{2} \int_{0}^{z_{H}} y^{3} e^{-3 A} d y-\int_{0}^{z_{H}} y^{3} e^{-3 A} e^{c y^{2}} d y\right)}\right.}{\left(1-e^{c z_{H}^{2}}\right)^{2}}\right]
$$

The temperature $T$ v.s. horizon $z_{H}$ at different chemical potentials is plotted in figure 2 . At $\mu=0$, the temperature has a global minimum $T_{\min }(0)$ at $z_{H}=z_{\min }(0)$. The black hole solution is only thermodynamically stable for $z_{H}<z_{\min }(0)$ and is unstable for $z_{H}>$ $z_{\min }(0)$. Below the temperature $T_{\min }(0)$, there is no black hole solution and we expect a Hawking-Page phase transition happens at a temperature $T_{\mathrm{HP}}(0) \gtrsim T_{\min }(0)$ where the black hole dissolves to a thermal gas background. For $0<\mu<\mu_{c}$, the temperature has a local minimum/maximum $T_{\min }(\mu) / T_{\max }(\mu)$ at $z_{H}=z_{\min }(\mu) / z_{\max }(\mu)$ and decreases to zero at a finite size of horizon. The black holes between $z_{\min }(\mu)$ and $z_{\max }(\mu)$ are thermodynamically unstable. There are two sections that are stable with $z_{H}<z_{\text {min }}(\mu)$ and $z_{H}>z_{\max }(\mu)$. We expect a similar Hawking-Page phase transition happens at a temperature $T_{\mathrm{HP}}(\mu) \gtrsim T_{\min }(\mu)$. Nevertheless, since the thermodynamically stable black hole solutions exist even when the temperature below $T_{\min }(\mu)$ for the section $z_{H}>z_{\max }(\mu)$, we also expect a black hole to black hole phase transition happening at a temperature $T_{B B}(\mu)$ between $T_{\min }(\mu)$ and $T_{\max }(\mu)$, where a large black hole with the horizon $z=$ $z_{H l}(\mu)$ collapses to a small black hole with the horizon $z=z_{H s}(\mu)$ as showed in figure 3. 


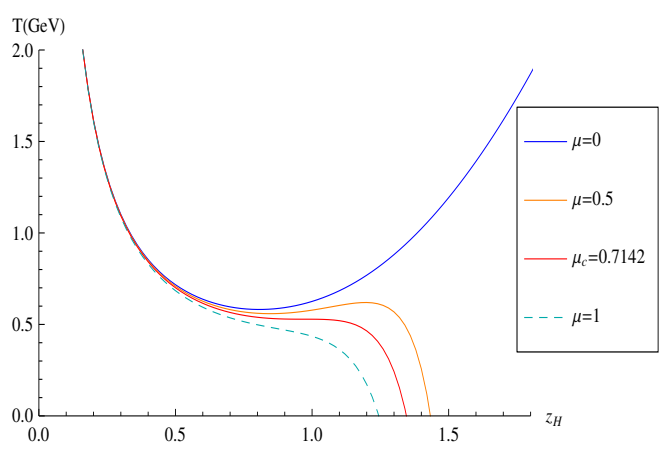

(a)

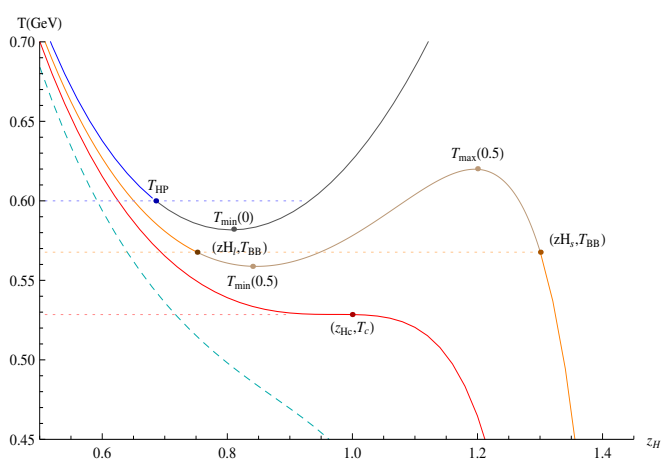

(b)

Figure 2. The temperature v.s. horizon at different chemical potentials $\mu=0,0.5,0.714,1 \mathrm{GeV}$. We enlarge a rectangle region in (a) into (b) to see the detailed structure. For $\mu>\mu_{c}$, the temperature decreases monotonously to zero; while for $\mu<\mu_{c}$, the temperature has a local minimum. At $\mu_{c} \simeq 0.714 \mathrm{GeV}$, the local minimum reduces to a inflection point.

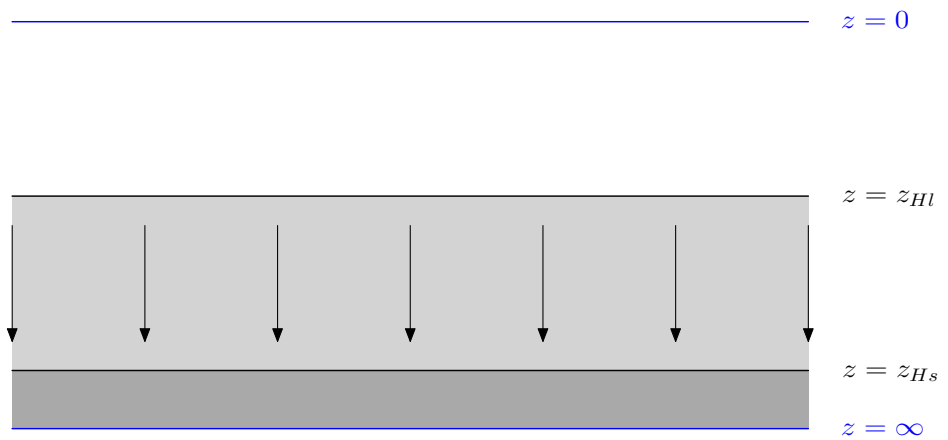

Figure 3. Phase transition from a large black hole with the horizon $z=z_{H l}$ collapses to a small black hole with the horizon $z=z_{H s}$ at the transition temperature $T=T_{B B}$.

Finally, for $\mu>\mu_{c}$, the temperature monotonously decreases to zero and there is no black hole to black hole phase transition anymore. ${ }^{1}$

To determine the phase transition temperatures $T_{\mathrm{HP}}(\mu)$ and $T_{B B}(\mu)$, we compute the free energy from the first law of thermodynamics in grand canonical ensemble

$$
F=-\int s d T
$$

We plot the free energy v.s. temperature in (a) of figure 4.

At $\mu=0$, the free energy intersect the $x$-axis at $T=T_{\mathrm{HP}}(0)$ where the Hawking-Page phase transition happens. The black hole dissolves to thermal gas which is thermodynamically stable for $T<T_{\mathrm{HP}}(0)$. We fix the parameter $b \simeq 0.273 G e V^{4}$ in eq. (2.17) by fitting the Hawking-Page phase transition temperature $T_{\mathrm{HP}}(0)$ with the lattice QCD simulation of $T_{\mathrm{HP}} \simeq 0.6 \mathrm{GeV}$ in [37].

\footnotetext{
${ }^{1}$ There could still be a Hawking-Page phase transition at some temperature for the case of $\mu>\mu_{c}$, but we will show later that the black hole solution is always thermodynamically favored in this case.
} 


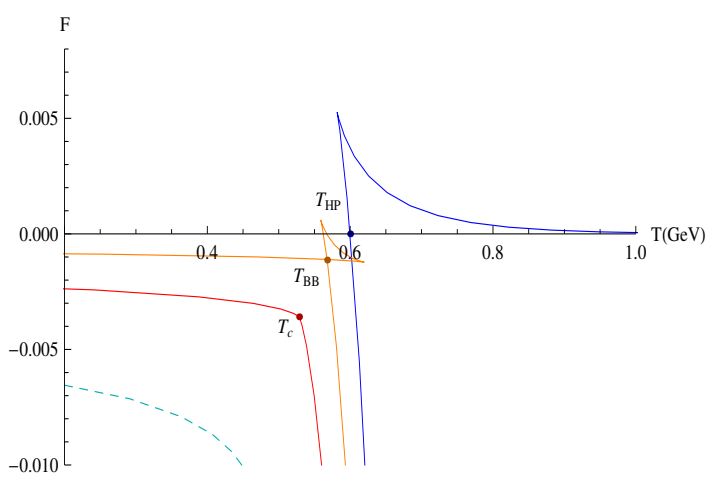

(a)

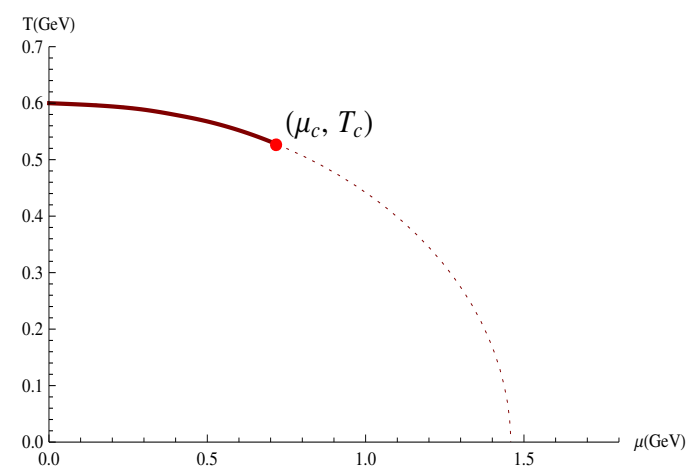

(b)

Figure 4. (a) The free energy v.s. temperature at chemical potentials $\mu=0,0.5,0.714,1 \mathrm{GeV}$. At $\mu=0$, the free energy intersect with the $x$-axis at $T=T_{\mathrm{HP}}$ where the black hole dissolves to thermal gas by Hawking-Page phase transition. For $0<\mu<\mu_{c} \simeq 0.714 \mathrm{GeV}$, the temperature reaches its maximum value where the free energy turns back to intersect with itself at $T=T_{B B}$ where the black hole to black hole transition happens. For $\mu>\mu_{c}$, the swallow-tailed shape disappears and there is no phase transition in the background. (b) The phase diagram in $T$ and $\mu$ plane. At small $\mu$, the system undergoes a first order phase transition at finite $T$. The first order phase transition stops at the critical point $\left(\mu_{c}, T_{c}\right)=(0.714 \mathrm{GeV}, 0.528 \mathrm{GeV})$, where the phase transition becomes second order. For $\mu>\mu_{c}$, the system weaken to a sharp but smooth crossover [20].

For $0<\mu<\mu_{c}$, the free energy behaves as the expected swallow-tailed shape. The temperature reaches its maximum where the free energy turns back and intersects with itself at $T=T_{B B}(\mu)$ where the large black hole transits to the small black hole. Since the free energies of the stable black holes are always less than that of the thermal gas $\left(F_{\text {gas }} \equiv 0\right)$, the thermodynamic system will always favor the small black hole background other than the thermal gas background. When we increase the chemical potential $\mu$ from zero to $\mu_{c}$, the loop of the swallow-tailed shape shrinks to disappear at $\mu=\mu_{c}$. For $\mu>\mu_{c}$, the curve of the free energy increases smoothly from higher temperature to lower temperature.

The phase diagram of the background is plotted in (b) of figure 4 . At $\mu=0$, the system undergoes a black hole to thermal gas phase transition at $T=T_{\mathrm{HP}}(0)$. For $0<\mu<\mu_{c}$, the system undergoes a large black hole to small black hole phase transition at $T_{B B}(\mu)$. The phase transition temperature $T_{B B}(\mu)$ approaches to $T_{\mathrm{HP}}$ at $\mu \rightarrow 0$ that makes the phase diagram continuous at $\mu=0$. The phase transition stops at $\mu=\mu_{c}$ and reduces to a crossover for $\mu>\mu_{c}$.

The phase diagram we obtained here in figure 4 is different from the conventional QCD phase diagram, in which crossover happens for small chemical potential and phase transition happens for large chemical potential. In [20], by comparing with the phase structure in lattice QCD simulation, the authors argued that this 'reversed' phase diagram should be interpreted as confinement-deconfinement phase transition of heavy quarks in QCD. In this paper, we consider the same background as in [20] to study pure gluon QCD with one additional heavy flavour, and not light quarks. Since our model describes heavy quarks system in QCD, the flavour field $A_{\mu}^{V}$ in the matter action 2.3 should be associated to the mesons make up of heavy quarks, i.e. quarkonium states. By fitting the lowest 


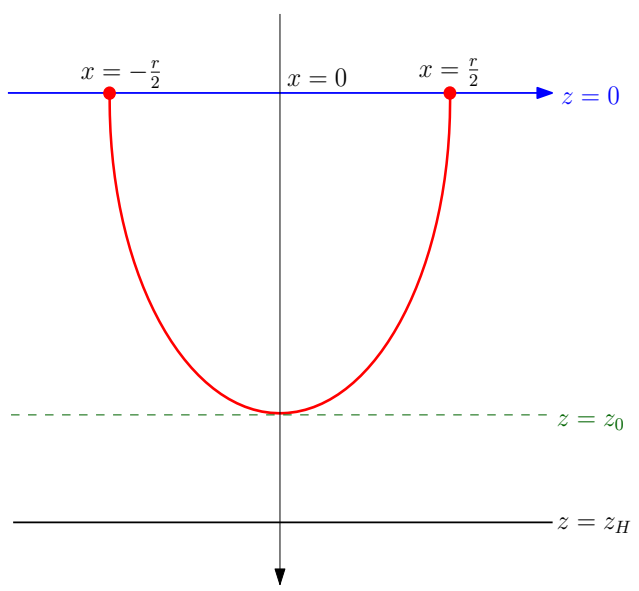

(a)

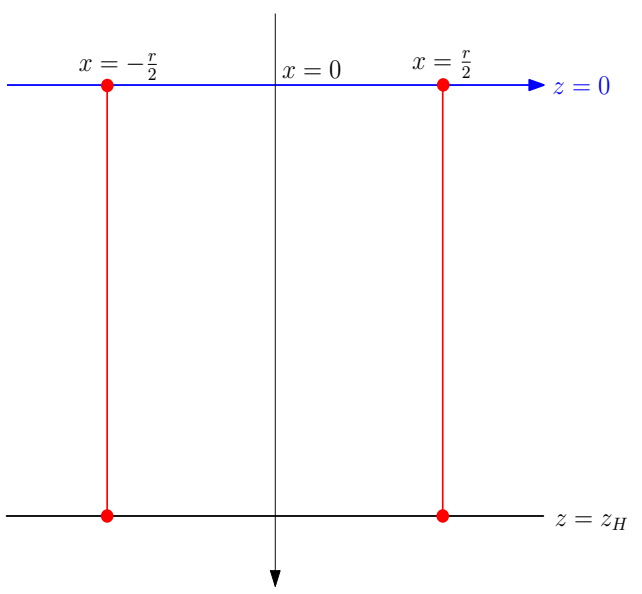

(b)

Figure 5. Two open string configurations. In (a), a U-shape open string connects its two ends at the boundary at $z=0$ and reaches its maximum depth at $z=z_{0}$. In (b), two straight open strings with their ends connecting the boundary at $z=0$ and the horizon at $z=z_{H}$, respectively.

two spectrum of quarkonium states $m_{J / \psi}=3.096 \mathrm{GeV}$ and $m_{\psi^{\prime}}=3.685 \mathrm{GeV}$, we can fix $c \simeq 1.16 \mathrm{GeV}^{2}$ in eq. (2.17).

Nevertheless, there left a problem that, in the gravity side, it is commonly believed that the confinement-deconfinement phase transition in the field theory side is dual to the Hawking-Page phase transition. Hawking-Page phase transition is the transition between black hole and thermal gas backgrounds. However, in our gravity background, the phase transition is between two black holes for a non-zero chemical potential. Thus it is not consistent to consider a black hole to black hole phase transition in the gravity side to be dual to the confinement-deconfinement phase transition in QCD. In the following of this paper, by adding open strings in the background, we will study this issue more carefully to gain a more reasonable physical picture.

\section{Open strings in the background}

In this paper, we consider an open string in the above background with its two ends on the boundary of the space-time at $z=0$. There are two configurations for an open string in the black hole background. One is the U-shape configuration with the open string reaching its maximum depth at $z=z_{0}$; the other is the straight configuration with the straight open string having its two ends attached to the boundary and the horizon at $z=z_{H}$, respectively. The two configurations are showed in figure 5. Since the dual holographic QCD lives on the boundary, it is natural for us to interpret the two ends of the open string as a quark-antiquark pair. The U-shape configuration corresponds to the quark-antiquark pair being connected by a string and can be identified as a meson state. While the straight configuration corresponds to a free quark or antiquark.

The Nambu-Goto action of an open string is

$$
S_{\mathrm{NG}}=\int d^{2} \xi \sqrt{-G}
$$




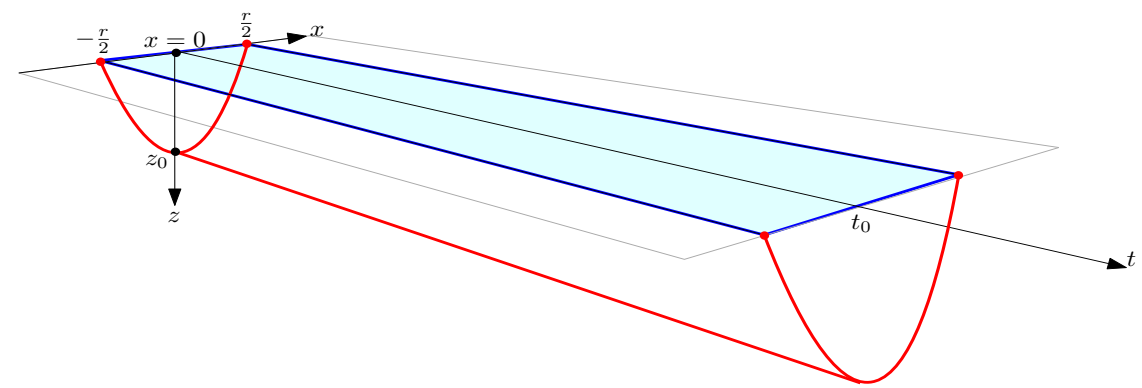

Figure 6. Wilson loop as the boundary of string world-sheet.

where the induced metric

$$
G_{a b}=g_{\mu \nu} \partial_{a} X^{\mu} \partial_{b} X^{\nu},
$$

on the 2-dimensional world-sheet that the string sweeps out as it moves with coordinates $\left(\xi^{0}, \xi^{1}\right)$ is the pullback of 5 -d target space-time metric $g_{\mu \nu}$,

$$
d s^{2}=\frac{e^{2 A(z)}}{z^{2}}\left(g(z) d t^{2}+d \vec{x}^{2}+\frac{1}{g(z)} d z^{2}\right),
$$

where, to study the thermal properties of the system, we consider the Euclidean metric and identify the periodic of the time with the inverse of temperature as $\beta=1 / T$.

\subsection{Wilson loop}

We consider a $r \times t_{0}$ rectangular Wilson loop $C$ along the directions $(t, x)$ on the boundary of the AdS space attached by a pair of the quark and antiquark separated by $r$. The quark and antiquark located at $(z=0, x= \pm r / 2)$ are connected by an open string, which reaches its maximum at $\left(z=z_{0}, x=0\right)$ as in figure 6 .

It is known that taking the limit $t_{0} \rightarrow \beta=1 / T$ allows one to read off the energy of such a pair from the expectation value of the Wilson loop,

$$
\langle W(C)\rangle \sim e^{-V(r, T) / T},
$$

where $V(r, T)$ is the heavy quark-antiquark potential [26, 33].

In string/gauge duality, the expectation value of the Wilson loop is given by

$$
\langle W(C)\rangle=\int D X e^{-S_{\mathrm{NG}}} \simeq e^{-S_{\mathrm{on}-\text { shell }}},
$$

where $S_{\mathrm{NG}}$ is the string world-sheet action bounded by a curve $C$ at the boundary of AdS space and $S_{\text {on-shell }}$ is the on-shell string action, which is proportional to the area of the string world-sheet bounded by the Wilson loop $C$.

Comparing with eq. (3.5), the free energy of the meson is defined as

$$
V(r, T)=T S_{\text {on-shell }}(r, T) .
$$




\subsection{Configurations of open strings}

The string world-sheet action is defined by the Nambu-Goto action,

$$
S=\int d^{2} \xi \mathcal{L}=\int d^{2} \xi \sqrt{\operatorname{det} G},
$$

where $G_{a b}=\partial_{a} X^{\mu} \partial_{b} X_{\mu}$ is the induced metric on string world-sheet. For the meson configuration, by choosing static gauge: $\xi^{0}=t, \xi^{1}=x$, the induced metric in string frame becomes

$$
d s^{2}=G_{a b} d \xi^{a} d \xi^{b}=\frac{e^{2 A(z)}}{z^{2}} g(z) d t^{2}+\frac{e^{2 A(z)}}{z^{2}}\left(1+\frac{z^{\prime 2}}{g(z)}\right) d x^{2},
$$

where the prime denotes a derivative with respect to $x$. The Lagrangian and Hamiltonian can be calculated as

$$
\begin{aligned}
\mathcal{L} & =\sqrt{\operatorname{det} G}=\frac{e^{2 A(z)}}{z^{2}} \sqrt{g(z)+z^{\prime 2}} \\
\mathcal{H} & =\left(\frac{\partial \mathcal{L}}{\partial z^{\prime}}\right) z^{\prime}-\mathcal{L}=-\frac{e^{2 A(z)} g(z)}{z^{2} \sqrt{g(z)+z^{\prime 2}}} .
\end{aligned}
$$

With boundary conditions

$$
z\left(x= \pm \frac{r}{2}\right)=0, z(x=0)=z_{0}, z^{\prime}(x=0)=0,
$$

we obtain the conserved energy

$$
\mathcal{H}(x=0)=-\frac{e^{2 A\left(z_{0}\right)}}{z_{0}^{2}} \sqrt{g\left(z_{0}\right)} .
$$

We can solve $z^{\prime}$ from eq. (3.10),

$$
z^{\prime}=\sqrt{g\left(\frac{\sigma^{2}(z)}{\sigma^{2}\left(z_{0}\right)}-1\right)}
$$

where

$$
\sigma(z)=\frac{e^{2 A(z)} \sqrt{g(z)}}{z^{2}} .
$$

The distance $r$ between the quark-antiquark pair can be calculated as,

$$
r=\int_{-\frac{r}{2}}^{\frac{r}{2}} d x=2 \int_{0}^{z_{0}} d z \frac{1}{z^{\prime}}=2 \int_{0}^{z_{0}} d z\left[g(z)\left(\frac{\sigma^{2}(z)}{\sigma^{2}\left(z_{0}\right)}-1\right)\right]^{-\frac{1}{2}}
$$

where $z_{0}$ is the maximum depth that the string can reach. The dependence of the distance $r$ on $z_{0}$ at two different horizons are plotted in figure 7 .

We see that for a small black hole (large $z_{H}$ ), there exist a dynamical wall at $z_{m}<z_{H}$ where $r^{\prime}\left(z_{m}\right) \rightarrow \infty$. The open string can not go beyond this dynamical wall, i.e. $z_{0} \leq z_{m}$, even when the distance $r$ between the quark-antiquark pair goes to infinity as showed in (a) of figure 8 . While for a large black hole ( small $z_{H}$ ), the open string can reach arbitrary close to the horizon, but there is a maximum value for the distance at $r=r_{M}$. If the 


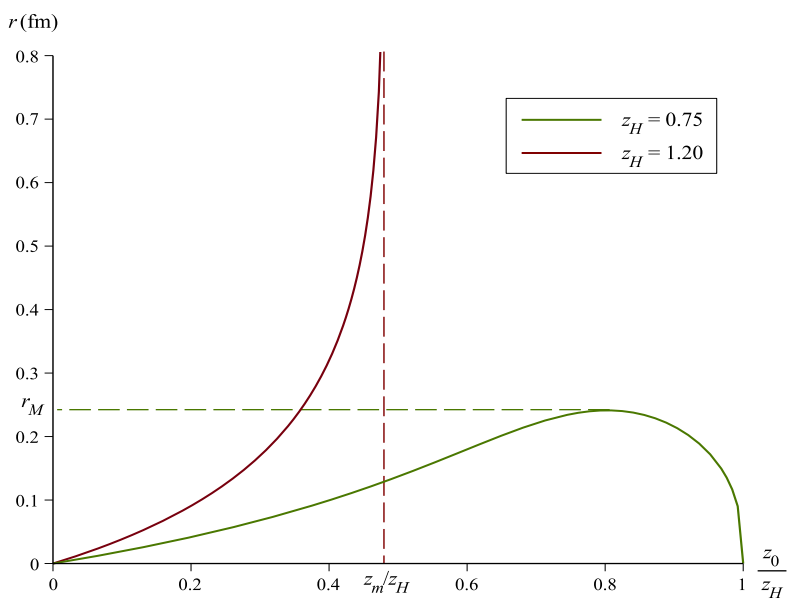

Figure 7. Separate distance $r$ between quark and antiquark v.s. $z_{0}$ at $\mu=0.5 \mathrm{GeV}$.
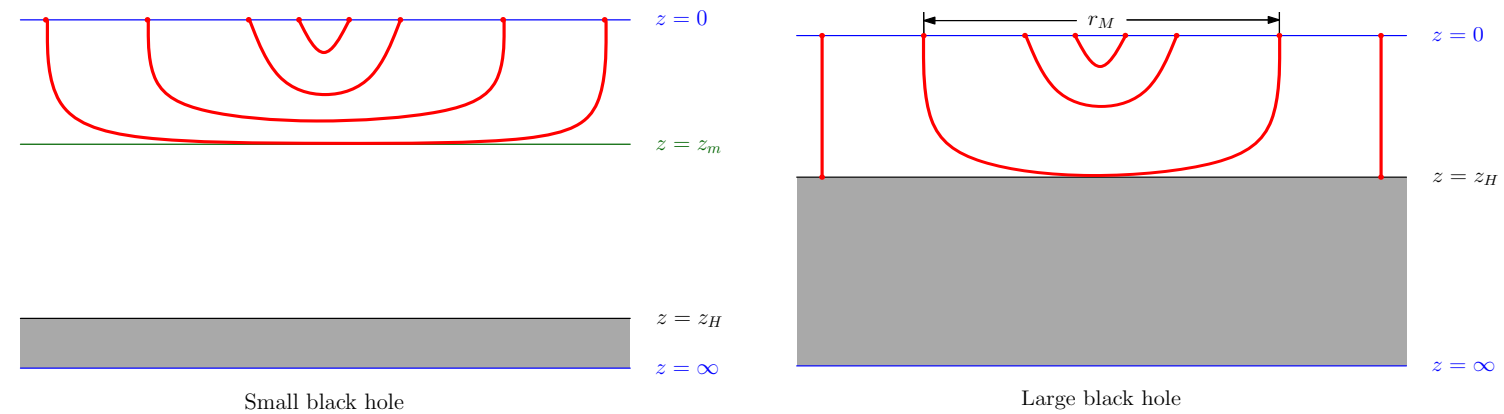

(b)

Figure 8. (a) For a small black hole, open strings can not exceed the dynamical wall at $z=z_{m}$ and are always in the U-shape. (b) For a large black hole, an open string will break to two straight strings if the distance between its two ends is larger than $z_{M}$.

distance between the quark and antiquark is larger than $r_{M}$, there is no stable U-shape solution for open strings so that the open string of U-shape will break to two straight open strings connecting the boundary at $z=0$ and the horizon at $z=z_{H}$ as showed in (b) of figure 8.

In summary, for a small black hole, open strings are always in the U-shape; while for a large black hole, an open string is in the U-shape for short separate distance $r<r_{M}$ and is in the straight shape for long separate distance $r>r_{M}$. Thus when a large black hole shrinks to a small one, we expect that a dynamical wall will appear when the black hole horizon equal to a critical value $z_{H \mu}$ for each chemical potential $\mu$, showed in figure 9 .

Since each horizon is associated to a temperatures for black holes, we define the transformation temperature $T_{\mu}$ corresponding to the critical black hole horizon $z_{H \mu}$, at which the dynamical wall appears/disappears, for each chemical potential $\mu$. The dependence of $T_{\mu}$ on $\mu$ is plotted in figure 10 .

When there is dynamical wall, open strings are always in the U-shape. This means that quarks and antiquarks are always connected by an open string to form a bound state, 


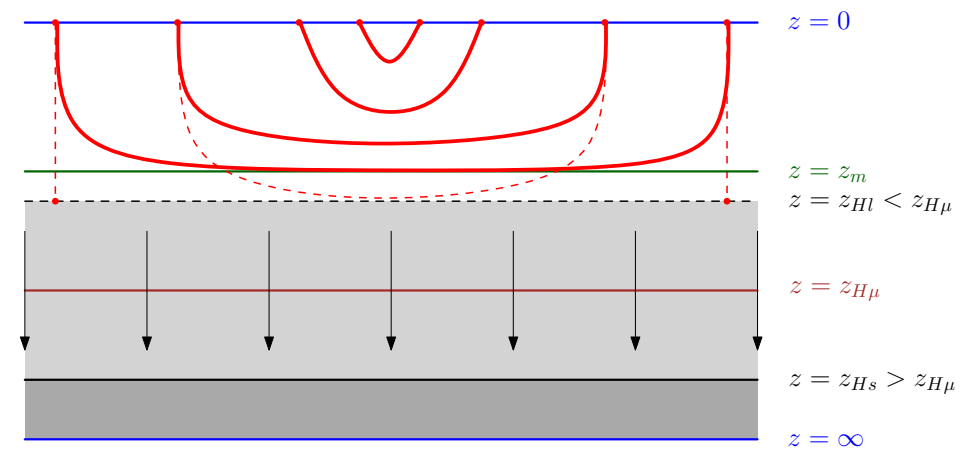

Figure 9. When a large black hole with horizon $z_{H l}<z_{H \mu}$ shrinks to a small black hole with horizon $z_{H s}>z_{H \mu}$, the dynamical wall at $z=z_{m}$ appears when $z_{H}=z_{H \mu}$.

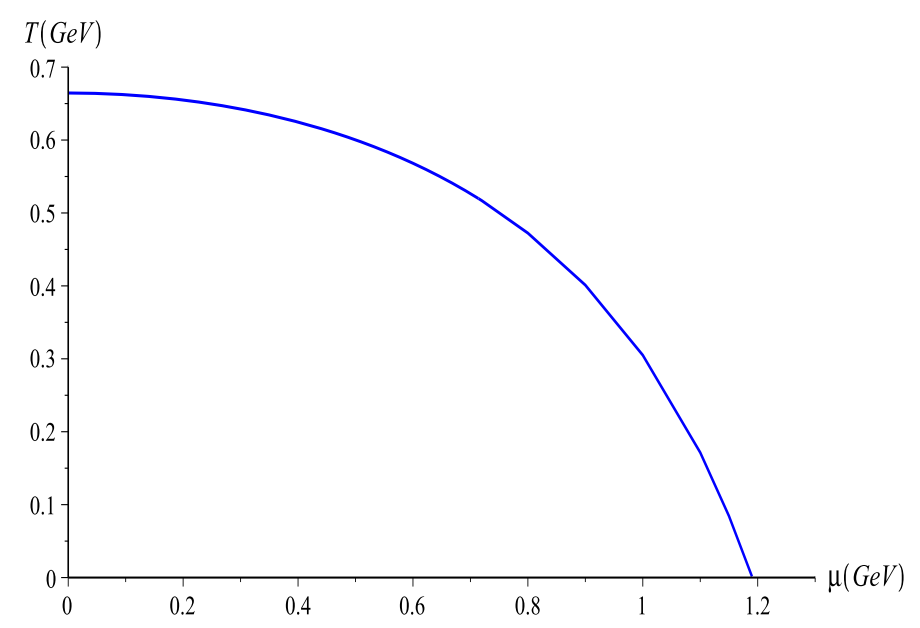

Figure 10. The temperature $T_{\mu}$ corresponding to $z_{H \mu}$, at which the dynamical wall appears/disaapears, for each chemical potential $\mu$.

i.e. the meson state in QCD. It is natural to interpret this case as the confinement phase in the dual holographic QCD. On the other hand, when the dynamical wall disappears, an Ushape open string could break up to two strings if the distance between its two ends is large enough. This means that a meson state could break to a pair of free quark and antiquark in QCD. We interpret this case as the deconfinement phase in the dual holographic QCD. Therefore, the transformation temperature $T_{\mu}$ is associated to the transformation between the confinement and the deconfinement phases in the dual holographic QCD. ${ }^{2}$

\subsection{Heavy quark potential}

When the black hole horizon $z_{H}>z_{H \mu}$, there exists a dynamical wall at $z=z_{m}<z_{H}$. Open strings are always in the U-shape and the heavy quark potential can be calculated as

$$
V=T S_{\text {on-shell }}=\int_{-\frac{r}{2}}^{\frac{r}{2}} d x \mathcal{L}=2 \int_{0}^{z_{0}} d z \frac{\sigma(z)}{\sqrt{g(z)}}\left[1-\frac{\sigma^{2}\left(z_{0}\right)}{\sigma^{2}(z)}\right]^{-\frac{1}{2}} .
$$

\footnotetext{
${ }^{2}$ This transformation between confinement and deconfinement phases is not necessary a phase transition, it could be a smooth crossover as we will show later.
} 
In short separate distance limit $r \rightarrow 0$, i.e. $z_{0} \rightarrow 0$, we expand the distance and the heavy quark potential at $z_{0}=0$,

$$
\begin{gathered}
r=2 \int_{0}^{z_{0}} d z\left[g(z)\left(\frac{\sigma^{2}(z)}{\sigma^{2}\left(z_{0}\right)}-1\right)\right]^{-\frac{1}{2}}=r_{1} z_{0}+O\left(z_{0}^{2}\right), \\
V=2 \int_{0}^{z_{0}} d z \frac{\sigma(z)}{\sqrt{g(z)}}\left[1-\frac{\sigma^{2}\left(z_{0}\right)}{\sigma^{2}(z)}\right]^{-\frac{1}{2}}=\frac{V_{-1}}{z_{0}}+O(1),
\end{gathered}
$$

where

$$
\begin{gathered}
r_{1}=2 \int_{0}^{1} d v\left(\frac{1}{v^{4}}-1\right)^{-\frac{1}{2}}=\frac{1}{2} B\left(\frac{3}{4}, \frac{1}{2}\right), \\
V_{-1}=2 \int_{0}^{1} \frac{d v}{v^{2}}\left(1-v^{4}\right)^{-\frac{1}{2}}=\frac{1}{2} B\left(-\frac{1}{4}, \frac{1}{2}\right) .
\end{gathered}
$$

This gives the expected Coulomb potential at short separate distance,

$$
V=-\frac{\kappa}{r}
$$

where

$$
\kappa=-\frac{1}{4} B\left(\frac{3}{4}, \frac{1}{2}\right) B\left(-\frac{1}{4}, \frac{1}{2}\right) \simeq 1.44 .
$$

In long separate distance $r \rightarrow \infty$, i.e. $z_{0} \rightarrow z_{m}$, we make a coordinate transformation $z=z_{0}-z_{0} w^{2}$. The distance $r$ and the heavy quark potential $V$ become

$$
\begin{gathered}
r=2 \int_{0}^{1} f_{r}(w) d w \\
V=2 \int_{0}^{1} f_{V}(w) d w
\end{gathered}
$$

where

$$
\begin{aligned}
& f_{r}(w)=2 z_{0} w\left[g\left(z_{0}-z_{0} w^{2}\right)\left(\frac{\sigma^{2}\left(z_{0}-z_{0} w^{2}\right)}{\sigma^{2}\left(z_{0}\right)}-1\right)\right]^{-\frac{1}{2}}, \\
& f_{V}(w)=2 z_{0} w \frac{\sigma\left(z_{0}-z_{0} w^{2}\right)}{\sqrt{g\left(z_{0}-z_{0} w^{2}\right)}}\left[1-\frac{\sigma^{2}\left(z_{0}\right)}{\sigma^{2}\left(z_{0}-z_{0} w^{2}\right)}\right]^{-\frac{1}{2}} .
\end{aligned}
$$

We learn from figure 7 that the distance $r$ is divergent at $z_{0}=z_{m}$, and the same happens for the heavy quark potential. By carefully analysis, we find that this divergence is due to the integrands $f_{r}(w)$ and $f_{r}(w)$ are divergent near the lower limit $w=0$, i.e. $z=z_{0} \rightarrow z_{m}$. To study the behaviors of the distance and the heavy quark potential at $z_{0}=z_{m}$, we expand $f_{r}(w)$ and $f_{V}(w)$ at $w=0$,

$$
\begin{aligned}
& f_{r}(w)=2 z_{0}\left[-2 z_{0} g\left(z_{0}\right) \frac{\sigma^{\prime}\left(z_{0}\right)}{\sigma\left(z_{0}\right)}\right]^{-\frac{1}{2}}+O(w), \\
& f_{V}(w)=2 z_{0} \sigma\left(z_{0}\right)\left[-2 z_{0} g\left(z_{0}\right) \frac{\sigma^{\prime}\left(z_{0}\right)}{\sigma\left(z_{0}\right)}\right]^{-\frac{1}{2}}+O(w) .
\end{aligned}
$$




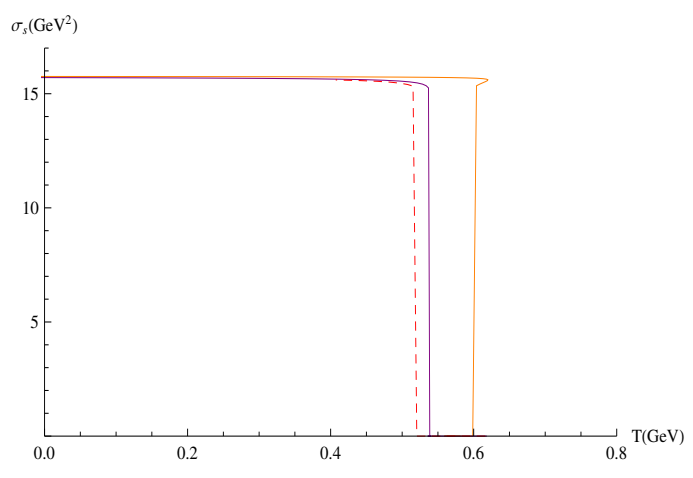

(a)

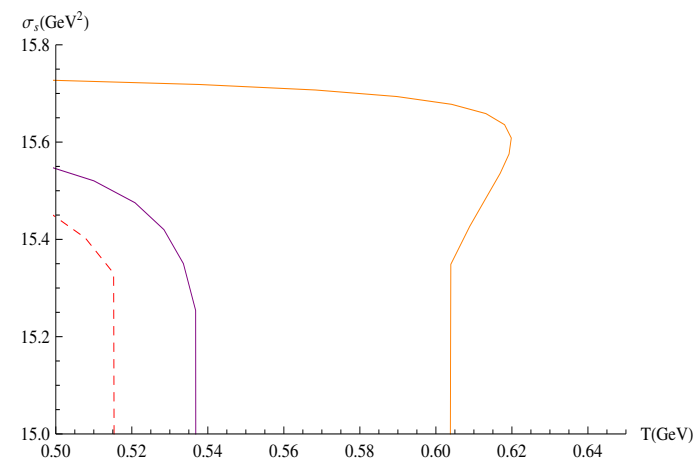

(b)

Figure 11. String tension v.s. temperature at $\mu=0.5,0.678,0.714 \mathrm{GeV}$. (a) The string tension decreases with temperature growing in the confinement phase, and suddenly drops to zero at $T=T_{\mu}$. The region closes to the transition temperatures is enlarged in (b).

The integrals (3.23) and (3.24) can be approximated by only consider the leading terms of $f_{r}(w)$ and $f_{r}(w)$ near $z_{0}=z_{m}$. This leads to

$$
\begin{aligned}
& r\left(z_{0}\right) \simeq 4 z_{0}\left[-2 z_{0} g\left(z_{0}\right) \frac{\sigma^{\prime}\left(z_{0}\right)}{\sigma\left(z_{0}\right)}\right]^{-\frac{1}{2}} \\
& V\left(z_{0}\right) \simeq 4 z_{0} \sigma\left(z_{0}\right)\left[-2 z_{0} g\left(z_{0}\right) \frac{\sigma^{\prime}\left(z_{0}\right)}{\sigma\left(z_{0}\right)}\right]^{-\frac{1}{2}}=\sigma\left(z_{0}\right) r\left(z_{0}\right) .
\end{aligned}
$$

From the above expression, we obtain the expected linear potential $V=\sigma_{s} r$ at long distance with the string tension,

$$
\sigma_{s}=\left.\frac{d V}{d r}\right|_{z_{0}=z_{m}}=\left.\frac{d V / d z_{0}}{d r / d z_{0}}\right|_{z_{0}=z_{m}}=\left.\frac{\sigma^{\prime}\left(z_{0}\right) r\left(z_{0}\right)+\sigma\left(z_{0}\right) r^{\prime}\left(z_{0}\right)}{r^{\prime}\left(z_{0}\right)}\right|_{z_{0}=z_{m}}=\sigma\left(z_{m}\right) .
$$

The temperature dependence of the string tension for various chemical potentials is plotted in figure 11. We see that the string tension decreases when the temperature increases. At the confinement-deconfinement transformation temperature $T_{\mu}$, the system transform to the deconfinement phase and the string tension suddenly drops to zero as we expected [38].

The behaviors of the heavy quark potential at short distance and long distance agrees with the form of the Cornell potential,

$$
V(r)=-\frac{\kappa}{r}+\sigma_{s} r+C
$$

which has been measured in great detail in lattice simulations

Next, we would like to look at the $r$ dependence of the heavy quark potential by evaluating the integral in eq. (3.16), which is divergent due to its integrand blows up at $z=0$. We simply regularize the integral by subtracting the divergent part of the integrand,

$$
V_{R}=C\left(z_{0}\right)+2 \int_{0}^{z_{0}} d z\left[\frac{\sigma(z)}{\sqrt{g(z)}}\left[1-\frac{\sigma^{2}\left(z_{0}\right)}{\sigma^{2}(z)}\right]^{-\frac{1}{2}}-\frac{1}{z^{2}}\left[1+2 A^{\prime}(0) z\right]\right]
$$




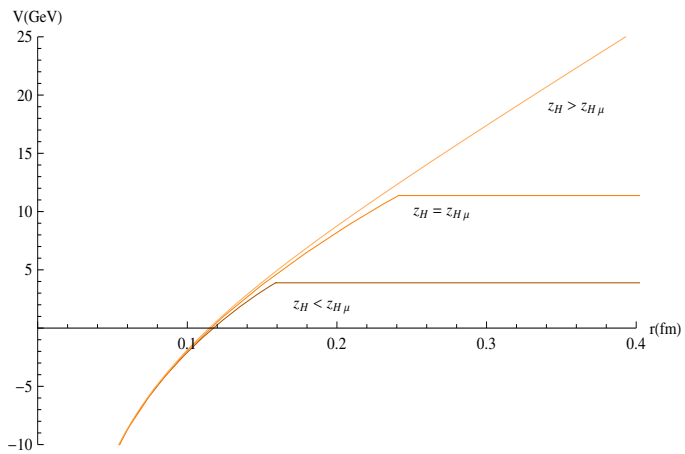

(a)

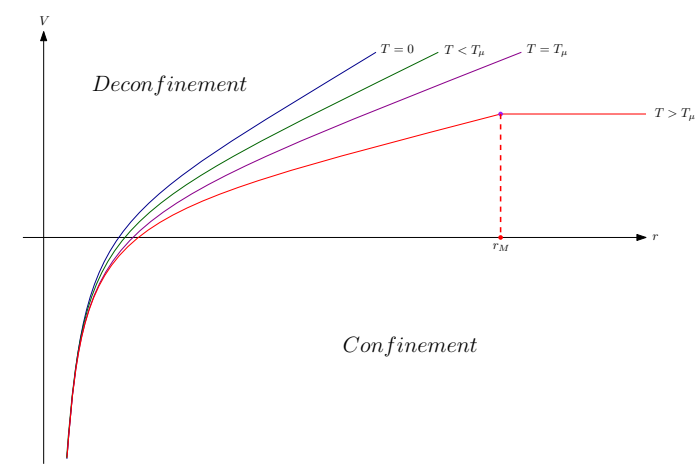

(b)

Figure 12. (a) $V$ v.s. $r$ at $\mu=0.5 \mathrm{GeV}$ for $z_{H}>z_{H \mu}, z_{H}=z_{H \mu}$ and $z_{H}<z_{H \mu}$. (b) The sketch diagram for heavy quark potentials at various temperatures at a fixed chemical potential $\mu>\mu_{c}$.

where

$$
C\left(z_{0}\right)=-\frac{2}{z_{0}}+4 A^{\prime}(0) \ln z_{0}
$$

After the regularization, we are able to calculate the heavy quark potential. The result is plotted in (a) of figure 12. For short separate distance, the potential is proportional to $1 / r$ as expected. While for long separate distance, there exists a critical horizon $z_{H \mu}$ for each chemical potential $\mu$. For small black hole with $z_{H}>z_{H \mu}$, the potential is linear to $r$ for $r \rightarrow \infty$. While for large black hole with $z_{H}<z_{H \mu}$, the potential ceases at a maximum distance $r_{M}$. Beyond $r_{M}$, the U-shape open string will break to two straight shape open strings.

It is helpful to use a sketch to describe the heavy quark potential and the phases transformation with temperature changing. We plot the heavy quark potentials at various temperatures at a fixed chemical potential ${ }^{3} \mu>\mu_{c}$ in (b) of figure 12. For $T \leq T_{\mu}$, i.e. $z_{H}>z_{H \mu}$, the heavy quark potential is linear at large separate distance $r$ with the slopes decrease with the temperature increasing. The linear potential implies that the system is in the confinement phase. While for $T>T_{\mu}$, i.e. $z_{H}<z_{H \mu}$, the heavy quark potential admits a maximum separate distance $r_{M}$, beyond which the open string breaks up to two straight strings and the total energy of strings becomes constant. The constant potential implies that the system is in the deconfinement phase. The confinement-deconfinement phase transform happens at $T=T_{\mu}$.

\section{Phase diagram}

In the previous sections, we have studied the thermodynamics of the black hole background. We obtained two black hole phases and studied the phase transition between them. We also added probe open strings in the background and studied their configurations of U-shape and straight shape, which correspond to confinement and deconfinement phases in the dual

\footnotetext{
${ }^{3}$ For $\mu<\mu_{c}$, the picture is similar but more complicated due to the black holes phase transition in the background. Here we just illustrate the general properties for the heavy quark potential and leave the details of the phase transition to the next section.
} 


\begin{tabular}{|c|c|c|}
\hline Black hole & String configurations for $r \rightarrow \infty$ & Phase in QCD \\
\hline Small $\left(z_{H}>z_{H \mu}\right)$ & U-shape & Confinement \\
\hline Large $\left(z_{H}<z_{H \mu}\right)$ & Straight & Deconfenment \\
\hline
\end{tabular}

Table 1. Black hole phases, open string configurations and QCD phases.

holographic QCD. In [20], we interpreted the black hole to black hole phase transition in the background as the confinement-deconfinement phase transition in the dual holographic QCD, leaving a puzzle that a black hole background does not correspond to the confinement phase in QCD in the original AdS/QCD correspondence. In this paper, we argued that the U-shape and straight shape of open strings should correspond to confinement and deconfinement phases in QCD, but the transformation between the two phases seems always smooth without phase transition. In this section, by combining these two phenomena, we are ready to discuss the full phase structure for the system of the open strings in the black hole background, corresponding to the confinement-deconfinement phase diagram in the dual holographic QCD.

\subsection{Confinement-deconfinement phase diagram}

Let us consider the configurations of the probe open strings first. We have found that for a small black hole with $z_{H}>z_{H \mu}$, open strings can not exceed a dynamical wall at $z=z_{m}$ even the distance $r$ between the quark-antiquark pair goes to infinity. This means that both ends of the open strings have to touch the boundary at $z=0$, and the quarkantiquark pair is always connected by an open string in the U-shape to form a bound state, which corresponds to a meson state in the dual holographic QCD, as in (a) of figure 8. We interpret this phase as the confinement phase in QCD. On the other hand, for a large black hole with $z_{H}<z_{H \mu}$, the two ends of the open strings could also contact the horizon instead of the boundary. If the distance $r$ between the quark-antiquark pair is large enough with $r>r_{M}$, an open string of U-shape would break to two straight open strings as showed in (b) of figure 8. Thus the meson state would decay to a pair of free quark and antiquark. We interpret this phase as the deconfinement phase in QCD. We should remark that for a small black hole with $z_{H}>z_{H \mu}$, it is impossible for an open string to break up due to the dynamical wall at $z=z_{m}$. Thus even in the black hole background, the holographic QCD could still be in the confinement phase. This clarifies the puzzle in [20] that a black hole background does not correspond to the confinement phase in QCD in the original AdS/QCD correspondence. The black hole phases, open string configurations and QCD phases are summarized in table 1 .

For each chemical potential $\mu$, we have calculated the transformation temperature $T_{\mu}$ corresponding to the critical black hole horizon $z_{H \mu}$. The result of $T_{\mu}$ v.s. $\mu$ is plotted in figure 10. On the other hand, the phase transition temperature $T_{B B}$ of black hole to black hole phase transition in the background was plotted in (b) of figure 4 . To investigate the relationship between $T_{\mu}$ and $T_{B B}$, we plot both of them together in (a) of figure 13. We see that the two lines are close to each other but not exactly the same. The two lines intersect 


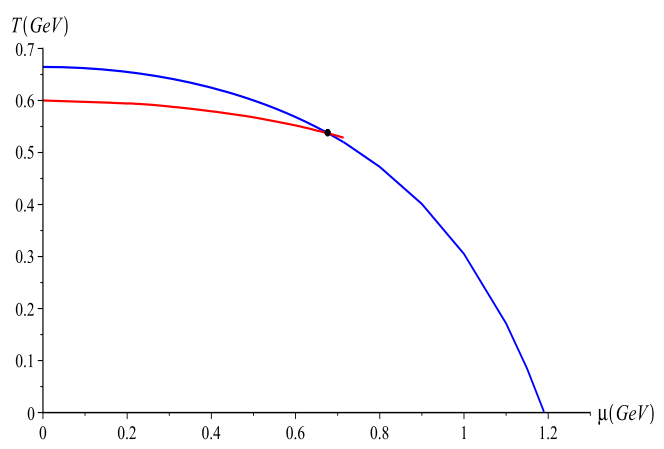

(a)

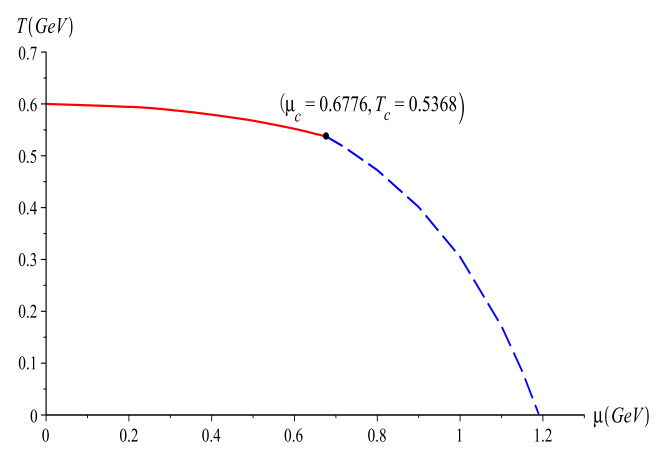

(b)

Figure 13. (a) The phase diagrams from the pure black hole background (red line for $T_{B B}$ ) and from the configurations of open strings (blue line for $T_{\mu}$ ). The two lines intersect at the critical point locates at $\mu_{c}=0.678 \mathrm{GeV}$ and $T_{c}=0.536 \mathrm{GeV}$. (b) The final confinement-deconfinement phase diagram. For $\mu<\mu_{c}$, there is a phase transition between confinement and deconfinement phases (red solid line); while for $\mu>\mu_{c}$, the phase transition becomes a crossover (blue dashed line).

at $\left(\mu_{c}, T_{c}\right)=(0.678 \mathrm{GeV}, 0.536 \mathrm{GeV})$, where we define as the critical point. ${ }^{4}$ For $\mu<\mu_{c}$, when the temperature increases from zero, the black hole grows with the temperature and a phase transition eventually happens at $T=T_{B B}(\mu)<T_{\mu}$, where a small black hole with horizon $z_{H s}>z_{H \mu}$ suddenly jumps to a large black hole with horizon $z_{H l}<z_{H \mu}$ as showed in figure 9. In the dual QCD, this implies that the confinement phase transform to the deconfinement phase by a phase transition. While for $\mu>\mu_{c}$, when the temperature increases from zero, the black hole horizon increases gradually with the temperature and continuously passes $z_{H \mu}$ at $T=T_{\mu}<T_{B B}(\mu)$. It means that the confinement phase will smoothly transform to the deconfinement phase as a crossover. Putting everything together, we obtain the final phase diagram for the confinement-deconfinement phase transition in QCD, plotted in (b) of figure 13. For the chemical potential less than the critical point $\mu<\mu_{c}$, we have confinement-deconfinement phase transition. While for the large chemical potential $\mu>\mu_{c}$, the confinement-deconfinement phase transition reduces to a smooth crossover. The critical point is located at $\mu_{c}=0.678 \mathrm{GeV}$ and $T_{c}=0.536 \mathrm{GeV}$. This result is consistent with the conclusion from the lattice QCD for the heavy quarks [37].

\subsection{Meson melting in hot plasma}

In the confinement phase, open strings are always connected in the configuration of Ushape, so that the quark-antiquark pair always form a bound state, i.e. a meson state in QCD. In the deconfinement phase, an open string could be in the configuration of either U-shape or straight-shape. For short separate distance $r<r_{M}$, the open string is still in the U-shape. While for the long separate distance $r>r_{M}$, the energy of the two straight

\footnotetext{
${ }^{4}$ We have defined $\left(\mu_{c}, T_{c}\right)=(0.714 \mathrm{GeV}, 0.528 \mathrm{GeV})$ as the critical values of the background phase transition in section 2.2 , here we redefined them as the true critical values of the confinement-deconfinement phase transition.
} 


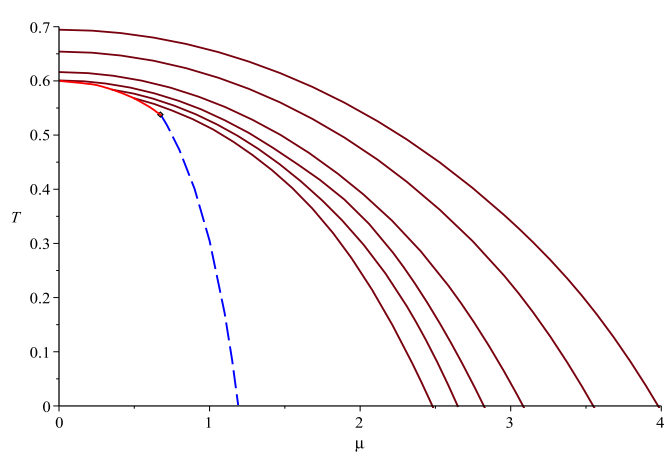

(a)

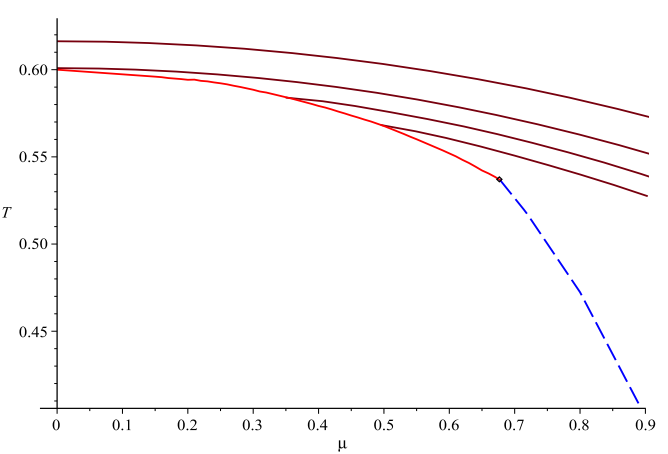

(b)

Figure 14. The 'melting lines' for various screening lengths are plotted in (a) with $r_{M}=$ $0.13,0.15,0.18,0.20,0.22,0.24 \mathrm{fm}$ from above. In (b), the region closed to the phase transition line is enlarged.

strings is less than the free energy of an open string in the U-shape and the U-shape open string will break up to two straight strings, corresponding to that a meson state melts to a pair of free quark and antiquark. The phenomenon of mesons melting has been previously studied in [38-41]. In this work, we define the screening length as the maximum length $r_{M}$, achieved by a pair of quark and antiquark in the bound state at a temperature $T>T_{B B}(\mu)$ for $\mu \leq \mu_{c}$ or $T>T_{\mu}$ for $\mu>\mu_{c}$. The screening length at a fixed chemical potential and temperature can be determined by the equation $V-2 F_{q}=0$, where $V$ is the heavy quark potential energy defined in (3.16) and $F_{q}$ is the free energy of a straight string defined as

$$
F_{q}=\int_{0}^{z_{H}} d z \frac{e^{2 A}}{z^{2}}
$$

We plot the 'melting lines' of screening length versus chemical potential in figure 14 . The screening length is a possible signal form Quark Gluon Plasma (QGP). Right after the collision QGP is formed and the temperature is high enough to be in the deconfinement phase. As temperature decreases, heavy quarks form bound states at melting temperatures higher than the deconfinement temperature. This means heavy quark bound sates can coexist with QGP.

\section{Conclusion}

In this paper, we considered an Einstein-Maxwell-scalar system. We solved the equations of motion to obtain a family of black hole solutions by potential reconstruction method. We studied the thermodynamical properties of the black hole background and found black hole to black hole phase transitions at the temperature $T_{B B}$ for the backgrounds. We then added open strings in these backgrounds and identified the two ends of an open string as a quark and antiquark pair in the dual holographic QCD. By solving the equations of motion of the open strings, we got two configurations for the open strings, i.e. Ushape and straight-shape. When the temperature is low enough, the black hole is small, there exists a dynamical wall at $z=z_{m}$ which the open strings can not exceed even the 
separation of the quark and antiquark goes to infinite. From the view of the dual QCD, the quark and antiquark pair is always connected by an open string to form a bounded state, corresponding to the confinement phase in QCD. On the other hand, when the temperature is high enough, the black hole becomes large so that an open string could break up to two straight open strings connecting the boundary and the black hole horizon, corresponding to the deconfinement phase in QCD. We obtained the confinement-deconfinement phase transformation temperature $T_{\mu}$.

Our main conclusion is that, to study the confinement-deconfinement phase structure in holographic QCD models, we need to combine two phase phenomena in the bulk gravity theory at the same time, namely the black hole to black hole phase transition in the background and the various configurations for the probe open strings. We found that, when the chemical potential is less than the critical value $\mu_{c}$, the background undergoes a small black hole to a large black hole phase transition with temperature increasing from zero. The horizon suddenly blows up to pass the critical horizon $z_{H \mu}$ so that the confinement phase transform to the deconfinement phase by a phase transition. While when the chemical potential is greater than the critical value $\mu_{c}$, the black hole horizon grows gradually and continuously pass the critical horizon $z_{H \mu}$ so that the confinement phase transform to the deconfinement phase by a smooth crossover. The final confinement-deconfinement phase diagram is showed in (b) of figure 13.

We also studied meson melting in this paper. When the temperature is higher than the phase transition temperature, QCD is in the deconfinement phase. However, it is known that the meson could still be thermodynamically stable in the deconfinement phase if the separate distance between the quark and antiquark is short enough. Only when the separate distance is longer than the screening length $r_{M}$, the meson becomes unstable and break up to a pair of free quark and antiquark. We showed the 'melting lines' for various separation distance in figure 14 . We conclude that, with increasing temperature, the mesons of larger size will break up earlier and the mesons of smaller size will be more stable and break up latter. Inversely, when QGP is cooling down, the mesons of smaller size will reunite earlier than the ones of larger size. This will help us to understand the process of the QGP cooling down.

\section{Acknowledgments}

We would like to thank Song He, Mei Huang, Xiao-Ning Wu for useful discussions. This work is supported by the National Science Council (NSC 101-2112-M-009-005) and National Center for Theoretical Science, Taiwan.

Open Access. This article is distributed under the terms of the Creative Commons Attribution License (CC-BY 4.0), which permits any use, distribution and reproduction in any medium, provided the original author(s) and source are credited.

\section{References}

[1] O. Philipsen, Lattice QCD at non-zero temperature and baryon density, arXiv:1009.4089 [INSPIRE]. 
[2] P. Petreczky, Lattice QCD at non-zero temperature, J. Phys. G 39 (2012) 093002 [arXiv:1203.5320].

[3] J. Babington, J. Erdmenger, N.J. Evans, Z. Guralnik and I. Kirsch, Chiral symmetry breaking and pions in nonsupersymmetric gauge/gravity duals, Phys. Rev. D 69 (2004) 066007 [hep-th/0306018] [INSPIRE].

[4] M. Kruczenski, D. Mateos, R.C. Myers and D.J. Winters, Towards a holographic dual of large- $N_{c} Q C D, J H E P 05$ (2004) 041 [hep-th/0311270] [INSPIRE].

[5] M. Kruczenski, D. Mateos, R.C. Myers and D.J. Winters, Meson spectroscopy in AdS/CFT with flavor, JHEP 07 (2003) 049 [hep-th/0304032] [INSPIRE].

[6] S. Kobayashi, D. Mateos, S. Matsuura, R.C. Myers and R.M. Thomson, Holographic phase transitions at finite baryon density, JHEP 02 (2007) 016 [hep-th/0611099] [INSPIRE].

[7] T. Sakai and S. Sugimoto, Low energy hadron physics in holographic QCD, Prog. Theor. Phys. 113 (2005) 843 [hep-th/0412141] [INSPIRE].

[8] T. Sakai and S. Sugimoto, More on a holographic dual of QCD, Prog. Theor. Phys. 114 (2005) 1083 [hep-th/0507073] [INSPIRE].

[9] J. Erlich, E. Katz, D.T. Son and M.A. Stephanov, QCD and a holographic model of hadrons, Phys. Rev. Lett. 95 (2005) 261602 [hep-ph/0501128] [INSPIRE].

[10] A. Karch, E. Katz, D.T. Son and M.A. Stephanov, Linear confinement and AdS/QCD, Phys. Rev. D 74 (2006) 015005 [hep-ph/0602229] [INSPIRE].

[11] B. Batell and T. Gherghetta, Dynamical soft-wall AdS/QCD, Phys. Rev. D 78 (2008) 026002 [arXiv: 0801.4383] [inSPIRE].

[12] W. de Paula, T. Frederico, H. Forkel and M. Beyer, Dynamical AdS/QCD with area-law confinement and linear Regge trajectories, Phys. Rev. D 79 (2009) 075019 [arXiv:0806.3830] [INSPIRE].

[13] S.S. Gubser and A. Nellore, Mimicking the QCD equation of state with a dual black hole, Phys. Rev. D 78 (2008) 086007 [arXiv: 0804.0434] [INSPIRE].

[14] C. Charmousis, B. Goutéraux, B.S. Kim, E. Kiritsis and R. Meyer, Effective holographic theories for low-temperature condensed matter systems, JHEP 11 (2010) 151 [arXiv: 1005.4690] [INSPIRE].

[15] U. Gürsoy, E. Kiritsis, L. Mazzanti, G. Michalogiorgakis and F. Nitti, Improved holographic QCD, Lect. Notes Phys. 828 (2011) 79 [arXiv: 1006.5461] [INSPIRE].

[16] O. DeWolfe, S.S. Gubser and C. Rosen, A holographic critical point, Phys. Rev. D 83 (2011) 086005 [arXiv: 1012.1864] [INSPIRE].

[17] D. Li, S. He, M. Huang and Q.-S. Yan, Thermodynamics of deformed AdS $S_{5}$ model with a positive/negative quadratic correction in graviton-dilaton system, JHEP 09 (2011) 041 [arXiv:1103.5389] [INSPIRE].

[18] O. DeWolfe, S.S. Gubser and C. Rosen, Dynamic critical phenomena at a holographic critical point, Phys. Rev. D 84 (2011) 126014 [arXiv:1108.2029] [InSPIRE].

[19] R.-G. Cai, S. Chakrabortty, S. He and L. Li, Some aspects of QGP phase in a hQCD model, JHEP 02 (2013) 068 [arXiv:1209.4512] [INSPIRE].

[20] S. He, S.-Y. Wu, Y. Yang and P.-H. Yuan, Phase structure in a dynamical soft-wall holographic QCD model, JHEP 04 (2013) 093 [arXiv: 1301.0385] [INSPIRE].

[21] G.S. Bali, QCD forces and heavy quark bound states, Phys. Rept. 343 (2001) 1 [hep-ph/0001312] [INSPIRE]. 
[22] E. Eichten et al., Charmonium: the model, Phys. Rev. D 17 (1978) 3090 [Phys. Rev. D 21 (1980) 203] [INSPIRE].

[23] J.M. Maldacena, Wilson loops in large N field theories, Phys. Rev. Lett. 80 (1998) 4859 [hep-th/9803002].

[24] S.-J. Rey, S. Theisen and J.-T. Yee, Wilson-Polyakov loop at finite temperature in large- $N$ gauge theory and anti-de Sitter supergravity, Nucl. Phys. B 527 (1998) 171 [hep-th/9803135] [INSPIRE].

[25] A. Brandhuber, N. Itzhaki, J. Sonnenschein and S. Yankielowicz, Wilson loops in the large N limit at finite temperature, Phys. Lett. B 434 (1998) 36 [hep-th/9803137] [INSPIRE].

[26] O. Andreev and V.I. Zakharov, Heavy-quark potentials and AdS/QCD, Phys. Rev. D 74 (2006) 025023 [hep-ph/0604204] [INSPIRE].

[27] H. Boschi-Filho and N.R.F. Braga, AdS/CFT correspondence and strong interactions, PoS (IC2006) 035.

[28] O. Andreev and V.I. Zakharov, On heavy-quark free energies, entropies, Polyakov loop and AdS/QCD, JHEP 04 (2007) 100 [hep-ph/0611304] [INSPIRE].

[29] C.D. White, The Cornell potential from general geometries in AdS/QCD, Phys. Lett. B 652 (2007) 79 [hep-ph/0701157] [INSPIRE].

[30] J.L. Albacete, Y.V. Kovchegov and A. Taliotis, Heavy quark potential at finite temperature in AdS/CFT revisited, Phys. Rev. D 78 (2008) 115007 [arXiv:0807.4747] [InSPIRE].

[31] S. He, M. Huang and Q.-S. Yan, Logarithmic correction in the deformed AdS $S_{5}$ model to produce the heavy quark potential and QCD $\beta$-function, Phys. Rev. D 83 (2011) 045034 [arXiv: 1004.1880] [INSPIRE].

[32] P. Colangelo, F. Giannuzzi and S. Nicotri, Holography, heavy-quark free energy and the QCD phase diagram, Phys. Rev. D 83 (2011) 035015 [arXiv:1008.3116] [INSPIRE].

[33] R.-G. Cai, S. He and D. Li, A hQCD model and its phase diagram in Einstein-Maxwell-Dilaton system, JHEP 03 (2012) 033 [arXiv:1201.0820] [INSPIRE].

[34] D. Li, M. Huang and Q.S. Yan, A dynamical soft-wall holographic QCD model for chiral symmetry breaking and linear confinement, Eur. Phys. J. C 73 (2013) 2615 [arXiv: 1206.2824] [INSPIRE].

[35] Y. Wu, D. Hou and H.C. Ren, Some comments on the holographic heavy quark potential in a thermal bath, arXiv:1401.3635 [INSPIRE].

[36] M. Shifman, Highly excited hadrons in QCD and beyond, hep-ph/0507246 [INSPIRE].

[37] M. Fromm, J. Langelage, S. Lottini and O. Philipsen, The QCD deconfinement transition for heavy quarks and all baryon chemical potentials, JHEP 01 (2012) 042 [arXiv:1111.4953] [INSPIRE].

[38] M. Mia, K. Dasgupta, C. Gale and S. Jeon, Heavy quarkonium melting in large- $N$ thermal QCD, Phys. Lett. B 694 (2011) 460 [arXiv: 1006.0055] [INSPIRE].

[39] M. Mia, K. Dasgupta, C. Gale and S. Jeon, A holographic model for large-N thermal QCD, J. Phys. G 39 (2012) 054004 [arXiv:1108. 0684] [INSPIRE].

[40] K. Fadafan, Heavy quarks in the presence of higher derivative corrections from AdS/CFT, Eur. Phys. J. C 71 (2011) 1799 [arXiv:1102.2289].

[41] K.B. Fadafan and E. Azimfard, On meson melting in the quark medium, Nucl. Phys. B $\mathbf{8 6 3}$ (2012) 347 [arXiv:1203.3942] [INSPIRE]. 